\title{
Sequential data fusion of GNSS Pseudoranges and Dopplers with map-based vision systems
}

\author{
Zui Tao and Philippe Bonnifait
}

\begin{abstract}
Tightly coupling GNSS pseudorange and Doppler measurements with other sensors is known to increase the accuracy and consistency of positioning information. Nowadays, high-accuracy geo-referenced lane marking maps are seen as key information sources in autonomous vehicle navigation. When an exteroceptive sensor such as a video camera or a lidar is used to detect them, lane markings provide positioning information which can be merged with GNSS data. In this paper, measurements from a forwards-looking video camera are merged with raw GNSS pseudoranges and Dopplers on visible satellites. To create a localization system that provides pose estimates with high availability, dead reckoning sensors are also integrated. The data fusion problem is then formulated as sequential filtering. A reduced-order state space modeling of the observation problem is proposed to give a real-time system that is easy to implement. A Kalman filter with measured input and correlated noises is developed using a suitable error model of the GNSS pseudoranges. Our experimental results show that this tightly coupled approach performs better, in terms of accuracy and consistency, than a loosely coupled method using GNSS fixes as inputs.
\end{abstract}

\section{INTRODUCTION}

Global Navigation Satellite Systems (GNSS) are widely used in vehicle navigation applications. There are two principal strategies for merging GNSS with other sources of information, namely loose coupling, which uses the GNSS fix computed by the receiver, and tight coupling which makes use of the raw GNSS observables (pseudorange, Doppler and carrier phase) on the visible satellites. Fig. 1 illustrates these two sensor fusion strategies.

Recently, there have been impressive demonstrations on both rural and urban routes of self-driving cars using production-based sensors and enhanced maps [39][16][30][29]. In most cases, an informative digital map makes autonomous driving possible using close-tomarket sensors. The creation of accurate digital maps for intelligent vehicles has seen significant progress [6][8] and these maps can now be produced on a large scale.

Using maps to assist and improve localization for autonomous navigation is a new paradigm in the research community, since it provides a way of using low-cost sensors. In particular, we are interested here in using low-cost monofrequency GNSS receivers (L1-receivers) that can use either GPS, Glonass or Galileo satellite constellations.

When using L1-GNSS with geo-referenced maps, experiments have shown that estimation methods need to estimate biases due to atmospheric effects, inaccurate satellite positions

The authors are with Sorbonne Universités, Université de Technologie de Compiègne, CNRS UMR 7253 Heudiasyc, 60200 Compiègne, France.

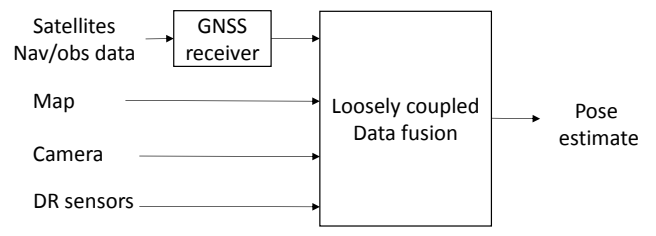

(a) Loosely coupled strategy. The estimates of the GNSS receiver are fed into the navigation filter.

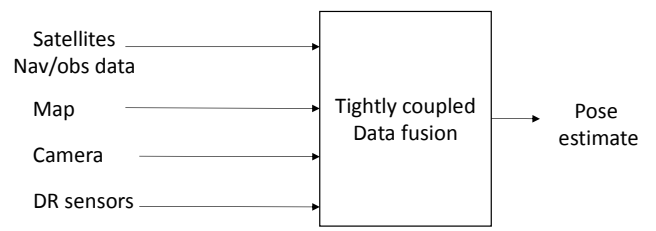

(b) Tightly coupled strategy with raw satellite measurements (pseudoranges, Dopplers).

Figure 1: Classical strategies for the fusion of GNSS data.

and mapping errors. In [19], the bias in GNSS fixes was estimated using the perception of lanes and crosswalks stored in the map. The authors of [21] proposed a localization method with GNSS error estimation based on curved lane detection and matching. In [34], different GNSS bias models were proposed and tested with a camera for detecting lane markings. This kind of approach can improve cross-track accuracy up to a decimeter-level. The along-track error is also controlled using heading variations in the trajectory [33]. However, loosely coupled methods depend on the performance of the GNSS receiver and on its software processing, which is particularly difficult to model.

This paper presents a tightly coupled scheme in which the bias on every pseudorange is estimated. When designing a localizer to access the pseudoranges, it is quite straightforward to obtain the Doppler measurements as well. Our proposed filter incorporates Dopplers, which are especially helpful for improving the performance of the positioning engine, since they give accurate range variation information. We use a forwards-looking camera that detects and localizes lane markings in the body frame of the car. By matching these detections with a geo-referenced map of the lane markings, we obtain redundant absolute positioning information. To get a high-frequency, high-availability pose for the car, the filter also utilizes wheel speed information and yaw rate from the ESP system installed on-board the car.

Another contribution of this paper is to compare the performance of this tightly coupled approach with a loosely coupled filter using real data. The criteria that we consider here are 
accuracy and consistency of estimation. We do not look at external integrity such as in [25] because although the filters reject outliers, they do not compute protection levels.

The remainder of this paper is organized as follows. Following a state of the art, the modeling of the map-aided localization system is given in section II. Section III introduces the GNSS measurement models and an extended Kalman filter with correlated noises (EKF-CN) is proposed for efficient embedded computation. Results of real outdoor experiments are presented and analyzed in section IV. In section V we present the performance metrics of our proposed tightly coupled method and compare them with a loosely coupled method. This final section also discusses the advantages and drawbacks of tight coupling with respect to loose coupling.

\section{STATE OF THE ART OF MAP-AIDED GNSS COMPUTATION}

Our research involves using maps of the road network. Since these maps are 2D with altitude information, they can be stored very compactly. The benefits of integrating a map in the computation of a position fix have been apparent for a long time. In [35], a Road Reduction filter was developed to calculate pseudorange corrections derived from the digital road network. Since no perception information is required, this macro-scale approach uses a pseudo measurement of the map by projecting the GNSS fix onto the road center-line. A navigation system incorporating this approach can achieve a horizontal position accuracy of $10 \mathrm{~m}(1 \sigma)$ [17]. It has been shown that uncertainty along the road can be further corrected by the pseudoranges whose line-of-sight directions are along the driving direction [14]. An alternative use of macro-scale maps is as a heading sensor, given that map precision is often better than map accuracy [15]. The height of the buildings on both sides of the road is a parameter that can be added to correct GNSS multipath using an urban trench model, as proposed by [5].

In order to take advantage of a high-accuracy map with a lane-level description of the carriageways (see [6] for instance) and to remove non-zero mean errors introduced by map matching on poly-lines, a vehicle needs to measure its relative position with respect to the lane which it is traveling in, by integrating perception information such as lane markings. In [32], a tightly coupled filter was described that combines raw GNSS measurements, camera observations and a lane marking map. Other recent works on tight coupling can be found in [27] and [18] with sophisticated pseudorange error models. In [27], pseudorange errors are estimated using Bayesian nonparametric noise models for multipath-contaminated signals. In [18], correlation errors are handled by a parallel cascade identification model. In [9], altitude-hold and clock bias prediction algorithms were proposed to provide a positioning solution in ill-conditioned situations. In the absence of an accurate map, vision sensors and differential corrections, the overall accuracy of these approaches remains inadequate for autonomous navigation.

Positioning accuracy is highly dependent on the accuracy of the pseudoranges. When integrating them with map-based vision measurements, a pseudorange $\rho^{i}$ is often modeled as:

$$
\rho^{i}=R^{i}+c \cdot d t_{u}+\varepsilon^{i}+\beta^{i}
$$

where $R^{i}$ is the geometrical distance between the receiver and the satellite, $d t_{u}$ the receiver clock offset, $c$ the speed of light, $\varepsilon^{i}$ a bias and $\beta^{i}$ the measurement noise for satellite $i$. $\varepsilon^{i}$ represents a common-mode error resulting from traversing the atmosphere and from ephemeris errors that affect the computed satellite positions. $\varepsilon^{i}$ can be removed by differential GNSS technology. $\beta^{i}$ is mainly caused by thermal noise and multipath.

The longitudinal vehicle speed is directly linked to Dopplers. In [36], the authors tightly coupled pseudoranges and Dopplers with vision measurements and Dead-Reckoning (DR) sensors to estimate vehicle 3D pose. They concluded that a single visual feature measurement at $1 \mathrm{~Hz}$ is able to achieve a submeter-level accuracy. In [28], a collaborative method was proposed for sharing geo-referenced vision measurements and GNSS pseudoranges to calibrate pseudorange errors for each satellite in view. In this method $\varepsilon^{i}$ is also modeled as a common-mode error. The advantage is that this method does not require a stationary reference receiver. The sharing of geo-referenced lane-boundary measurements can make $\varepsilon^{i}$ fully solvable (in the least-squares sense) for networked GNSS receivers. Using the lane-boundary measurements it is only possible to estimate the cross-track part of $\varepsilon^{i}$ in the road. In order to make the pseudorange errors fully observable, a collaborative method was proposed to estimate the correlated biases $\varepsilon^{i}$ among different rovers which are on different roads and can observe different parts of the error. As discussed in [11], sharing satellite measurements in a vehicular network also improves the heading estimation.

For a standalone receiver, the number of unknowns $\varepsilon^{i}$ (pseudorange bias) grows linearly with the number of measurements. However, it should be remembered that the biases $\varepsilon^{i}$ are time-correlated. If a good model can be established [3], then it is possible to estimate them using a filter. For instance, a stationary first-order autoregressive process driven by zeromean white noise $w_{\varepsilon}$ was given in [12]:

$$
\left\{\begin{array}{l}
\varepsilon_{k+1}^{i}=\lambda \varepsilon_{k}^{i}+w_{\varepsilon} \\
\lambda=e^{-T / \tau_{p r}}
\end{array}\right.
$$

where $T$ is the sampling period.

This kind of shaping filter is often used in practice. For instance, a time constant $\tau_{p r}=600$ s was suggested in [24]. It should be remarked that when implementing first-order shaping filters, the dimension of the state of the filter is increased by the number of visible satellites, because each pseudorange requires a shaping filter. Often, the observability of $\varepsilon^{i}$ is made possible by using DR sensors. Below we present a method where the estimation of these unknowns depends mainly on map-based vision measurements.

\section{LANE MARKING AIDED LOCALIZATION}

In this work, we consider a map of road markings made up of dashed or solid lane markings in the center or on the sides of the roads. If the vehicle knows approximately where it is located in the map, it is possible to implement a localization 


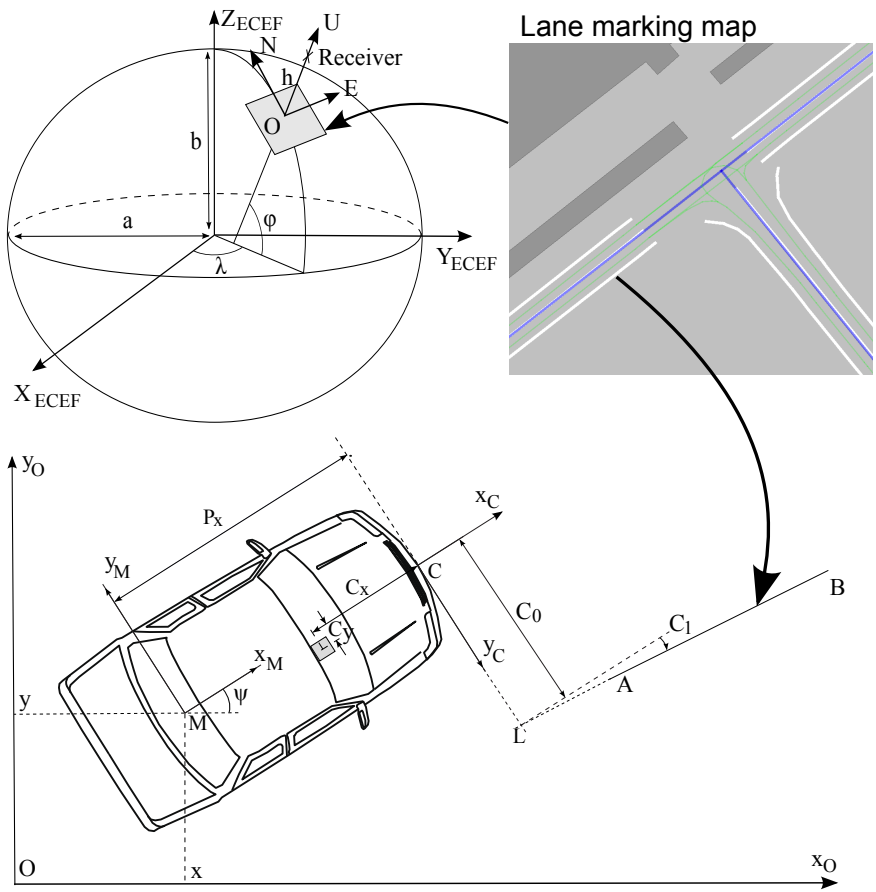

Figure 2: Frames: ECEF for GNSS, $R_{O}$ ENU for local navigation, $R_{M}$ for the mobile frame and $R_{C}$ for the camera.

The upper plot shows a zoomed part of the map. Lane marking polylines are plotted in white. In blue, the polylines of the carriageways. In green, the center of the lanes.

method that retrieves the absolute position and corrects the drift in DR estimates [23]. In the present section we outline the key concepts in implementing such an approach with visionbased lane marking measurements.

\section{A. Frames}

GNSS receivers usually use ECEF (Earth-Centered, EarthFixed) coordinates $(X, Y, Z)$ and provide geographic data $(\lambda, \varphi, h)$ (longitude, latitude, ellipsoid height). See Fig. 2.

For road vehicle navigation, a local East, North, Up (ENU) frame with Cartesian coordinates is more convenient for computing distances and angles and for modeling the system. An ENU frame (denoted $R_{O}$ below) is defined to be tangent to the Earth ellipsoid at a chosen origin. The North axis is tangent to the meridian that contains the origin and in the North direction. The East axis is normal to the North axis and is in the positive longitudes direction. The Up axis is chosen so as to yield a right-handed coordinate system. When the navigation area is reasonably horizontal and if it is close to the origin of $R_{O}$, only 2D coordinates (East and North) need to be considered.

Lane markings are expressed by polylines (Fig. 2). The lane marking map is geo-referenced in the World Geodetic System WGS84 with geographical coordinates. A local map corresponding to the navigation area is extracted and converted in $R_{O}$. When the car approaches a border of the local map, a swap is performed to refocus the estimate [7].

Two more frames are necessary. $R_{M}$ denotes the mobile vehicle reference frame $\left(x_{M}\right.$ is the along-track axis pointing forwards and $y_{M}$ is such that $z_{M}$ is upwards). Point $C$, the origin of the camera frame $R_{C}$, is located at the front of the vehicle. In order to stay consistent with vision system conventions, $y_{C}$ is right-hand oriented. Even if the camera is located behind the windscreen with a position offset $\left(C_{x}, C_{y}\right)$, every detected lane marking is expressed in $R_{C}$.

Let $\mathbf{q}=(x, y, \psi)^{T}$ be the pose of the vehicle. Unless otherwise specified, the coordinates are expressed in the working frame $R_{O} . \mathbf{q}$ includes the position $(x, y)$ and the heading angle $\psi$ with respect to the East.

\section{B. Kinematic model for dead reckoning}

The linear velocity of each rear wheel is measured by speed sensors used by the ABS system. As the experimental vehicle is front-wheel drive, we may assume that the rear wheels have a negligible slip. The speed vector is then collinear with axis $x_{M}$ which conducts to the unicycle model:

$$
\left\{\begin{array}{l}
\dot{x}=v^{m} \cdot \cos \psi \\
\dot{y}=v^{m} \cdot \sin \psi \\
\dot{\psi}=\omega^{m}-\varepsilon_{\omega} \\
\dot{\varepsilon}_{\omega}=0
\end{array}\right.
$$

where the linear velocity is given by $v^{m}=\left(v_{r l}^{m}+v_{r r}^{m}\right) / 2$. The measurement noise is denoted by $\gamma^{v} . v_{r l}^{m}$ and $v_{r r}^{m}$ are the measured linear velocity of the left and right rear wheels respectively. The angular velocity of the vehicle $\omega^{m}$ is measured by the ESP yaw rate gyro with a measurement noise $\gamma^{\omega} . \varepsilon_{\omega}$ is the gyro bias modeled by a random constant. An estimate of the pose $\mathbf{q}$ of the vehicle is obtained by integrating these measurements from a known initial pose.

\section{Camera measurement}

Let $L$ denote the lane marking detection located at ordinate $C_{0}$ in $R_{C}$ (Fig. 2). Its coordinates are given by:

$$
\left[\begin{array}{l}
x_{L} \\
y_{L}
\end{array}\right]=\left[\begin{array}{c}
P_{x} \cdot \cos \psi+C_{0} \cdot \sin \psi+x \\
P_{x} \cdot \sin \psi-C_{0} \cdot \cos \psi+y
\end{array}\right]
$$

$P_{x}$ refers to the translation between point $M$ and the bumper. Let $[\mathrm{AB}]$ be the detected lane marking segment with coordinates $\left(x_{A}, y_{A}\right)$ and $\left(x_{B}, y_{B}\right)$. Let $x_{A B}=x_{B}-x_{A}$ and $y_{A B}=y_{B}-y_{A}$.

Since $L$ is on segment $[A B]$, we have with $\lambda \in[0,1]$ :

$$
\left\{\begin{array}{l}
x_{L}=x_{A}+\lambda \cdot x_{A B} \\
y_{L}=y_{A}+\lambda \cdot y_{A B}
\end{array}\right.
$$

Plugging Eq. (4) into Eq. (5), we get:

$$
\left\{\begin{array}{c}
P_{x} \cdot \cos \psi+C_{0} \cdot \sin \psi+x=x_{A}+\lambda \cdot x_{A B} \\
P_{x} \cdot \sin \psi-C_{0} \cdot \cos \psi+y=y_{A}+\lambda \cdot y_{A B}
\end{array}\right.
$$

Since $x_{A B} \cdot \cos \psi+y_{A B} \cdot \sin \psi \neq 0$ (the detected segment is not perpendicular to the vehicle), the camera observation model becomes:

$$
C_{0}=\frac{\left(P_{x} \cdot \sin \psi+y-y_{A}\right) x_{A B}-\left(P_{x} \cdot \cos \psi+x-x_{A}\right) y_{A B}}{x_{A B} \cdot \cos \psi+y_{A B} \cdot \sin \psi}
$$

\section{Map-matching}

The problem is to determine which is the lane marking segment $[A B]$ that the camera has detected. The map-matching 


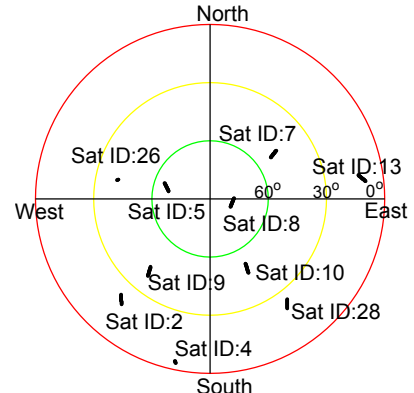

(a) Satellites configuration

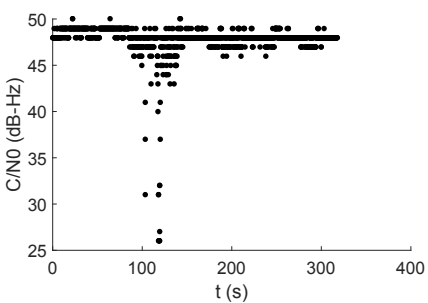

(b) $C / N_{0}$ over time for satellite \#8
Figure 3: $C / N_{0}$ variation for the highest satellite during test 1.

procedure that is used here combines metric information and symbolic information. The marking type (e.g. dashed or solid) given by the video camera is used to map-match the detected lane marking segment. This is done in two steps. In the first step a set $S$ of candidate segments is selected based on the following conditions:

- The lane marking type is consistent with that estimated by the camera,

- The orientation of the segment is close to the heading of the vehicle,

- The distance $d$ between point $L$ and the candidate segment is less than the width of the road.

In the second step the segment $s$ which has the smallest distance $\mathrm{d}$ is chosen as the map-matching result:

$$
\text { Map_matched }=\arg \min _{s \in\{S\}}\{\mathrm{d}\}
$$

\section{Tightly COUPLing L1-GNSS}

\section{A. GNSS measurements used in a tightly coupled scheme}

An L1-GNSS receiver tracks all the satellites in view. It provides raw measurements (C/A pseudorange, Doppler and signal-to-noise ratio - $C / N_{0}$ ) with respect to each GPS satellite in view. A GNSS receiver has a delay lock loop (DLL) for code tracking and a phase lock loop (PLL) for carrier phase tracking. The measurements produced by the DLL are the code pseudoranges. The Doppler shift is computed by tracking the frequency of the received signal and its value is immune to cycle slips [2]. $C / N_{0}$ refers to the ratio of the carrier power to the noise power per unit bandwidth [10].

Navigation information makes it possible to retrieve positions, velocities, and clock offsets of the satellites at their emission time. Common disturbances (such as ionosphere, relativity effects, etc.) are usually corrected using broadcast GNSS data and models.

When a satellite appears on the horizon, $C / N_{0}$ is in the range of $30-40 \mathrm{~dB}-\mathrm{Hz}$ and increases up to a maximum of slightly over $50 \mathrm{~dB}-\mathrm{Hz}$ once the satellite is at 50-60 degrees elevation angle or higher. As shown in Fig. 3a, there were a total of 10 satellites in view over our outdoor experimental area. Take the highest satellite (satellite 8) as an example. Fig. $3 \mathrm{~b}$ displays the variation of its $C / N_{0}$ over time. From around $\mathrm{t}=120 \mathrm{~s}$, the vehicle was traveling through an urban area and the $C / N_{0}$ degraded rapidly during this period. Low $C / N_{0}$ can also arise because of unintentional interference, deliberate jamming, or weak signals [17].

\section{B. System modeling}

For an accurate computation, the lever arm $\left[t_{x}, t_{y}, t_{z}\right]^{T}$ of the GNSS antenna with respect to the body frame has to be taken into account. The position $\mathbf{x}_{a}=\left[x_{a}, y_{a}, z_{a}\right]^{T}$ of the receiver antenna is given by:

$$
\left\{\begin{array}{l}
x_{a}=x+\cos \psi \cdot t_{x}-\sin \psi \cdot t_{y} \\
y_{a}=y+\sin \psi \cdot t_{x}+\cos \psi \cdot t_{y} \\
z_{a}=z+t_{z}
\end{array}\right.
$$

Note that the 3D position of the vehicle must be used. For a given satellite $i$, its position vector $\mathbf{x}_{s}^{i}=\left[x^{i}, y^{i}, z^{i}\right]^{T}$ at the emission time is reconstructed from the navigation message. The corresponding pseudorange is:

$$
\rho^{i}=\sqrt{\left(x_{a}-x^{i}\right)^{2}+\left(y_{a}-z^{i}\right)^{2}+\left(z_{a}-z^{i}\right)^{2}}+c \cdot d t_{u}+\varepsilon^{i}+\beta^{i}
$$

where $\varepsilon^{i}$ represents the residual (non-white) errors of the pseudorange and $\beta^{i}$ is the measurement noise.

The Doppler shift is caused by the relative motion of the satellite with respect to the receiver antenna. The line-of-sight vector $u_{\text {los }}^{i}$ of satellite $i$ is:

$$
u_{\text {los }}^{i}=\left(\mathbf{x}_{a}-\mathbf{x}_{s}^{i}\right) / R^{i}
$$

The Doppler shift is linked to the unknown via the following equation:

$$
\dot{\rho}^{i}=\left(\mathbf{v}_{r}-\mathbf{v}_{s}^{i}\right) \bullet u_{l o s}^{i}+c \cdot d \dot{t}_{u}+\dot{\varepsilon}^{i}+\beta_{d}^{i}
$$

where $\mathbf{v}_{r}=\left[v^{m} \cdot \cos \psi, v^{m} \cdot \sin \psi, 0\right]^{T}$ is the velocity vector of the receiver and $\mathbf{v}_{s}^{i}=\left[\dot{x^{i}}, \dot{y}^{i}, \dot{z}^{i}\right]^{T}$ that of satellite $i$. $\bullet$ denotes the dot product. $\beta_{d}^{i}$ is the measurement noise. $d \dot{t}_{u}$ is the drift of the clock of the receiver. The impact of the vehicle angular speed on the Doppler effect due to the antenna lever arm is neglected. Compared to a classical Doppler observation model, the derivative of the non-white pseudorange errors in Eq. (12) is taken into account.

The filter estimates range equivalent-values for the receiver clock parameters, and so we define the following variables:

$$
d=c \cdot d t_{u} \quad \dot{d}=c \cdot \dot{d t_{u}}
$$

If the altitude changes slowly in the working frame, it can be considered constant and estimated using altitude maps [26]. The Up coordinate $z_{\text {lane }}$ of the lane segment stored in the map can also be used (after matching the estimated 2D position) to compute the altitude $z_{a}$ :

$$
z_{a}=h_{a}+z_{\text {lane }}
$$

where $h_{a}$ is the height of the GNSS antenna with respect to the road. Fig. 4 shows that the path used in the experiments was almost planar.

There are three main sources of range errors (cf. Fig. 5): 1) atmospheric pseudorange residual errors $\varepsilon_{p r}, 2$ ) inaccurate satellite position estimates due to the use of real-time navigation messages $\varepsilon_{\text {Sat }}$ and 3) errors in the mapped altitude of the $\operatorname{road} \varepsilon_{z}$. 

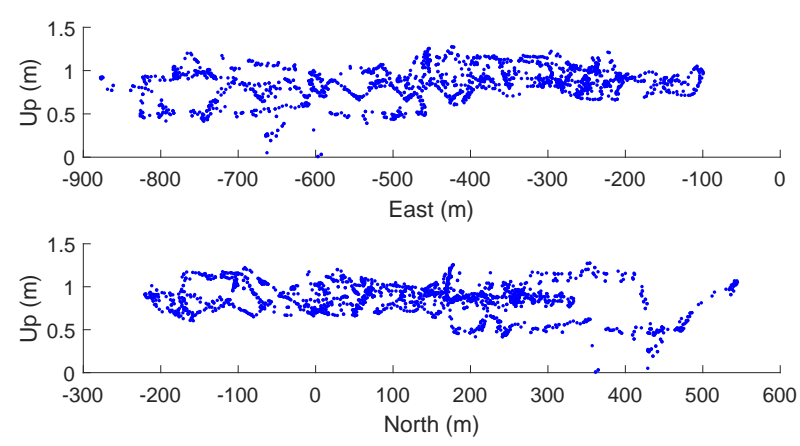

Figure 4: Up ordinates of nodes and shape points of the map.

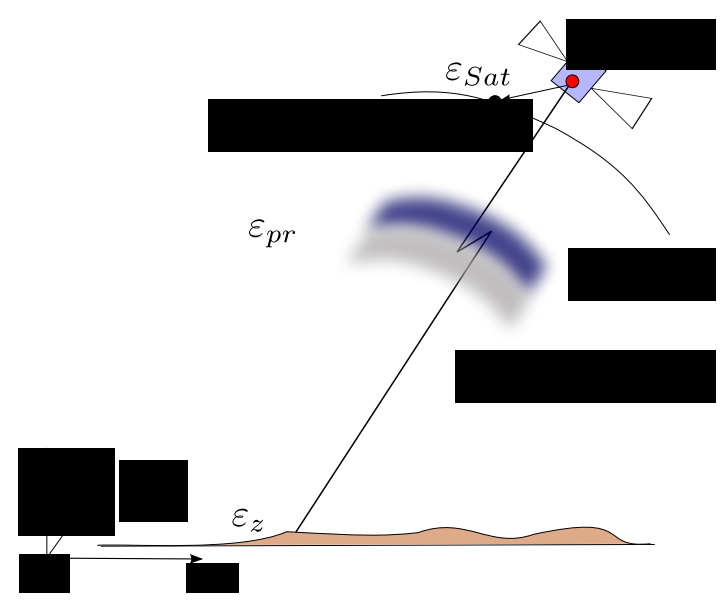

Figure 5: Range-error sources.

We propose estimating a range-error parameter $\varepsilon$ that results from the combination of $\varepsilon_{S a t}, \varepsilon_{p r}$ and $\varepsilon_{z}$. A first-order autoregressive shaping filter with driving noise $\beta^{\varepsilon i}$ is given by:

$$
\dot{\varepsilon}^{i}=-\varepsilon^{i} / \tau+\beta^{\varepsilon i}
$$

The time constant $\tau$ is the same for every satellite. The model given in Eq. (15) is used to estimate the derivative in Eq. (12).

With $n$ satellites in view, the state vector $\mathbf{x}$ becomes:

$$
\mathbf{x}=\left[x, y, \psi, \varepsilon_{\omega}, d, \dot{d}, \varepsilon^{1}, \cdots, \varepsilon^{n}\right]^{T}
$$

With the 2D kinematic model of Eq. (3), a discrete evolution model is given by the following (the subscript $k$ corresponding to the time stamp):

$$
\begin{aligned}
& \mathbf{x}_{k}=f\left(\mathbf{x}_{k-1}, \mathbf{u}_{k}^{m}, \boldsymbol{\alpha}_{k}\right) \\
& \boldsymbol{\alpha}_{k}=\left[\begin{array}{llllll}
\alpha_{k}^{\omega} & \alpha_{k}^{d} & \alpha_{k}^{d} & \alpha_{k}^{\varepsilon 1} & \ldots & \alpha_{k}^{\varepsilon n}
\end{array}\right]^{T} \\
& \Leftrightarrow\left\{\begin{array}{l}
x_{k}=x_{k-1}+T \cdot v_{k}^{m} \cdot \cos \psi_{k-1} \\
y_{k}=y_{k-1}+T \cdot v_{k}^{m} \cdot \sin \psi_{k-1} \\
\psi_{k}=\psi_{k-1}+T \cdot\left(w_{k}^{m}-\varepsilon_{\omega, k-1}\right) \\
\varepsilon_{\omega, k}=\varepsilon_{\omega, k-1}+\alpha_{k}^{\omega} \\
d_{k}=d_{k-1}+T \cdot \dot{d}_{k-1}+\alpha_{k}^{d} \\
\dot{d}_{k}=\dot{d}_{k-1}+\alpha_{k}^{\dot{d}} \\
\varepsilon_{k}^{1}=e^{-T / \tau} \varepsilon_{k-1}^{1}+\alpha_{k}^{\varepsilon 1} \\
\vdots \\
\vdots \\
\varepsilon_{k}^{n}=e^{-T / \tau} \varepsilon_{k-1}^{n}+\alpha_{k}^{\varepsilon n}
\end{array}\right.
\end{aligned}
$$

where $\mathbf{u}^{m}=\left[v^{m}, \omega^{m}\right]^{T}$ denotes the measured input vector and $T$ the sampling period. The wheel speed sensor measurement noise $v_{v}$ and the gyro measurement noise $v_{\omega}$ are assumed to be zero-mean white noise. $\mathbf{N}$ denotes their covariance matrix. $\alpha_{k}^{\varepsilon i}$ is driving noise of the shaping filter for pseudorange bias. The covariance of the model noise $\boldsymbol{\alpha}_{k}$ of Eq. (18) is denoted by Q. It will be noted that the pose uncertainty arises as a result of the uncertainty on the input.

\section{Outlier rejection}

A Kalman filter is sensitive to outliers resulting from multipath on buildings, for instance. There are several improvements that can be made to robustify a Kalman filter [1]. Other approaches harness new robust methods used in image processing [38] to reject outliers before the Kalman updates.

The method that we use here is to test the Dopplers and then the pseudoranges at every estimation stage. At time instant $k$ when GNSS measurements are available, a validation step is performed on every satellite measurement to avoid using badly tracked satellites and to reject multipath signals.

For Dopplers, the validation process involves checking the three three conditions:

- $C / N_{0}$ gating: Check that $C / N_{0}$ is high enough (e.g. 38 $\mathrm{dBHz}$.

- Elevation mask: The elevation angle of satellite $i$ is computed using $\mathbf{x}_{s}^{i}$ and the current estimate $\mathbf{x}_{k}$. The elevation mask angle is usually set to 15 degrees.

- Innovation gating using Normalized Innovation Squared (NIS).

When a Doppler is an outlier, then the corresponding pseudorange is in general an outlier [20]. But, even if a Doppler measurement is valid, the corresponding pseudorange can be faulty. So innovation gating needs to be done on the pseudorange after testing the Dopplers.

\section{Filter implementation}

The tightly coupled filter is described in Algorithm 1. The process is time-triggered with the CAN bus data which has the highest rate.

Since the filter uses a measured linear velocity $v^{m}$ with a noise $\gamma^{v}$ in the Doppler observation model (see Eq. (12)), the estimation process has correlated noises and the filter needs to be reformulated as an EKF with correlated noise (EKF-CN) [22]. When using Doppler measurements to update the state vector, the Kalman gain $\mathbf{K}$ is calculated as:

$$
\begin{aligned}
& \mathbf{N}=\operatorname{Var}\left(\gamma^{v}\right) \\
& \mathbf{B}=\frac{\partial f\left(\mathbf{x}_{k-1}, \mathbf{u}_{k}^{m}\right)}{\partial v^{m}} \\
& \mathbf{D}=\frac{\partial \dot{\rho}^{i}\left(\mathbf{x}_{k-1}, \mathbf{u}_{k}^{m}\right)}{\partial v^{m}} \\
& \mathbf{H}=\frac{\partial \dot{\rho}^{i}\left(\mathbf{x}_{k-1}, \mathbf{u}_{k}^{m}\right)}{\partial \mathbf{x}} \\
& \mathbf{S}=\mathbf{B} \cdot \mathbf{N} \cdot \mathbf{D}^{T} \\
& \mathbf{M}=\mathbf{H} \cdot \mathbf{P} \cdot \mathbf{H}^{T}+\mathbf{D} \cdot \mathbf{N} \cdot \mathbf{D}^{T}+\mathbf{R}+\mathbf{H} \cdot \mathbf{S}+\mathbf{S}^{T} \cdot \mathbf{H}^{T} \\
& \mathbf{K}=\left(\mathbf{P} \cdot \mathbf{H}^{T}+\mathbf{S}\right) \cdot \mathbf{M}^{-1}
\end{aligned}
$$

The update of the covariance in this case is:

$$
\mathbf{P}=\mathbf{P}-\mathbf{K}\left(\mathbf{H} \cdot \mathbf{P}+\mathbf{S}^{T}\right)
$$




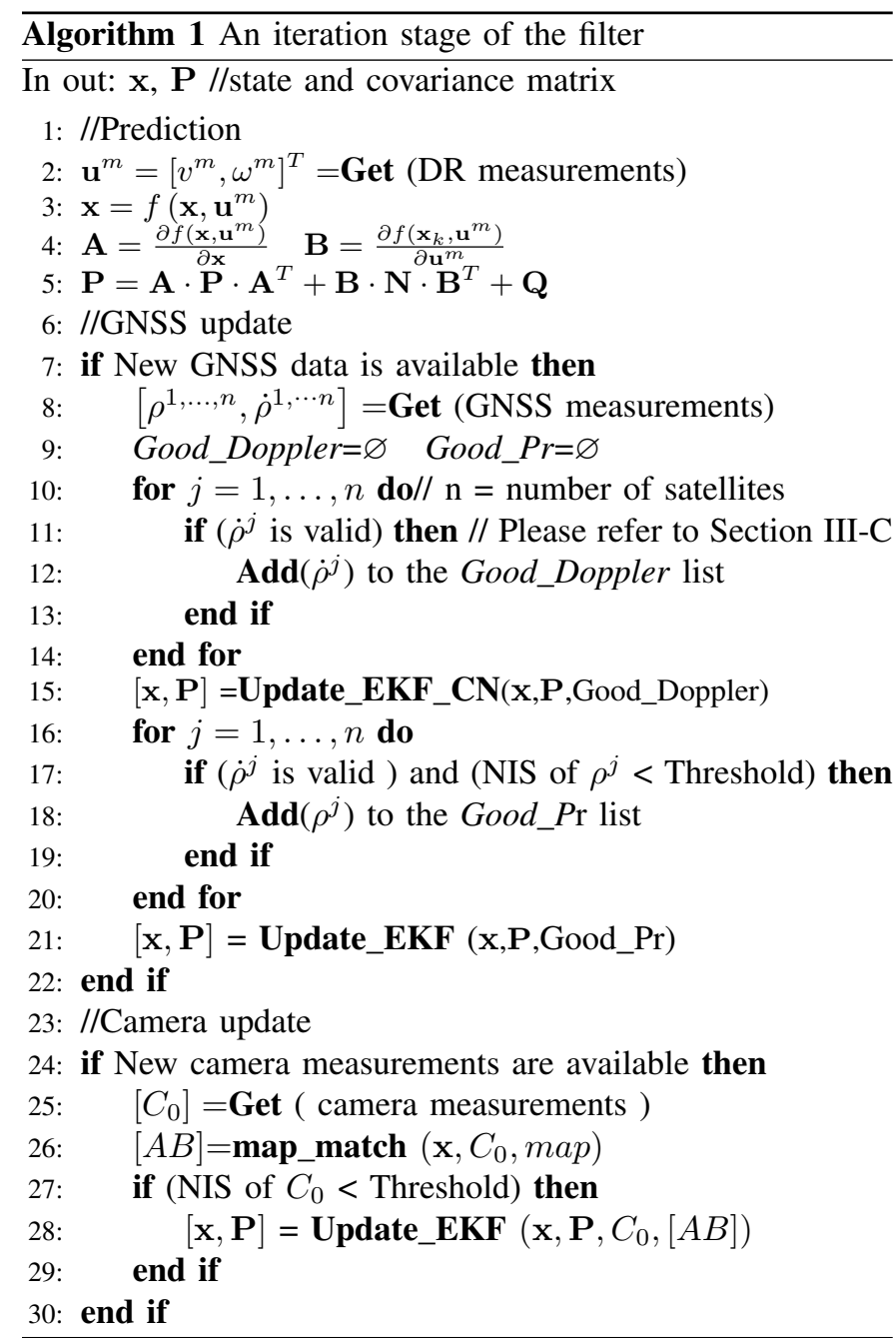

This way of updating the state of the filter is designated "Update_EKF_CN" in Algorithm 1.

In the algorithm, "Update_EKF" means a classical EKF update. As the camera sometimes provides detections that are done using distant markings and that can be very inaccurate, an NIS test on $C_{0}$ is implemented to reject those measurements before updating the state vector. The latency of the camera measurements has been compensated for in the results presented here, since we used time-stamps with a $1 \mathrm{~ms}$ accuracy. Moreover, as the camera provides what are essentially lateral corrections, and since here the speed of car is less than 30 $\mathrm{km} / \mathrm{h}$, latency errors of this order of magnitude have almost no impact on the accuracy.

\section{E. Parameter tuning}

The measurement noise $\beta^{i}$ in Eq. (10) is assumed to be white, zero mean and with a known variance $\left(\beta^{i} \sim N\left(0, \sigma_{i}^{2}\right)\right)$. As its variance is not stationary, we use Wieser's model [37] in which the variance depends on the measured carrier-to-noise density ratio $C / N_{0}$ :

$$
\sigma_{i}^{2}=S \cdot 10^{\frac{-C / N_{0}^{i}}{10}}
$$

with $S=60000 m^{2} H z$.

\begin{tabular}{|c|c|}
\hline Model noises variances & Measurement noises variances \\
\hline $\operatorname{Var}\left(\alpha^{\omega}\right)=5 \times 10^{-10}$ & $\operatorname{Var}\left(\beta_{c}\right)=0.16$ \\
$\operatorname{Var}\left(\alpha^{d}\right)=1 \times 10^{-3}$ & $\operatorname{Var}(\beta)=60000 \cdot 10^{\frac{-C / N_{0}^{i}}{10}}$ \\
$\operatorname{Var}\left(\alpha^{d}\right)=1 \times 10^{-4}$ & $\operatorname{Var}\left(\beta_{d}\right)=0.05$ \\
$\operatorname{Var}\left(\alpha^{\varepsilon}\right)=1 \times 10^{-4}$ & $\operatorname{Var}\left(\gamma^{v}\right)=1 \times 10^{-4}$ \\
$\operatorname{Var}\left(\gamma^{\omega}\right)=2.5 \times 10^{-3}$ \\
\hline
\end{tabular}

Table I: EKF-CN parameters (International System Units)

The other parameters of the filter were tuned using stationary observations for the gyro and trial-and-error tests. The time constant $\tau$ of Eq. (19) was set to 80 s, as suggested by Le Marchand in [20]. Table I indicates the values used in the filter for the process and measurement noises.

\section{EXPERIMENTAL RESULTS}

\section{A. Experimental set-up}

To evaluate the proposed method, we post-processed with Matlab some real data from outdoor experiments carried out near Paris, France. An experimental car was equipped with an IMU Oxford RT3000 which provided ground truth data at a rate of $100 \mathrm{~Hz}$. A CAN-bus gateway was used to access the wheel speed sensors and the yaw rate gyro. The CAN data was available at $100 \mathrm{~Hz}$. A Mobileye camera was installed behind the windscreen to detect the lane markings at $10 \mathrm{~Hz}$. A u-blox 6T GPS receiver with a patch antenna provided raw measurements at $5 \mathrm{~Hz}$. The ephemeris and range corrections were decoded and computed using the GPSTK library [4].

Three tests were performed on the same road with an experimental automotive vehicle in urban conditions. The traveling distance for each test was about $2 \mathrm{~km}$, with a typical speed of $30 \mathrm{~km} / \mathrm{h}$. In Fig. 6, the red line represents the traveled path during the first trial. The boxes in grey are buildings. Blue lines are mapped lane markings. The vehicle started at $\mathrm{t}=$ $0 \mathrm{~s}$ and stopped at $\mathrm{t}=327 \mathrm{~s}$. Between $\mathrm{t}=80 \mathrm{~s}$ and $\mathrm{t}=140 \mathrm{~s}$, the vehicle was going through a strong urban canyon of 300 meters and the U-blox receiver suffered from multipath around $\mathrm{t}=123 \mathrm{~s}$.

\section{B. Assessment of accuracy}

Fig. 7 shows the along-track and cross-track errors (in a Frenet frame) with estimated $3 \sigma$ bounds. The filter is well consistent and the along-track confidence bound decreases after the first right turn. The highest errors occur at crossroads when there is no lane detection.

Fig. 8 shows the altitudes of the GNSS antenna extracted from the map. The discrete shape is clearly visible.

Fig. 9 displays the heading errors which are less than 2 degrees and very consistent. Good estimates of the heading angle $\psi$ are important for implementing effective control laws [31].

In Fig. 10 it can be seen that the estimated receiver clock bias and the corresponding $3 \sigma$ bounds of the estimation errors are convergent.

Fig. 11 shows the estimated receiver clock bias drift and the $3 \sigma$ bounds of the estimation errors. Both clock bias and clock bias drift have converging uncertainties. 


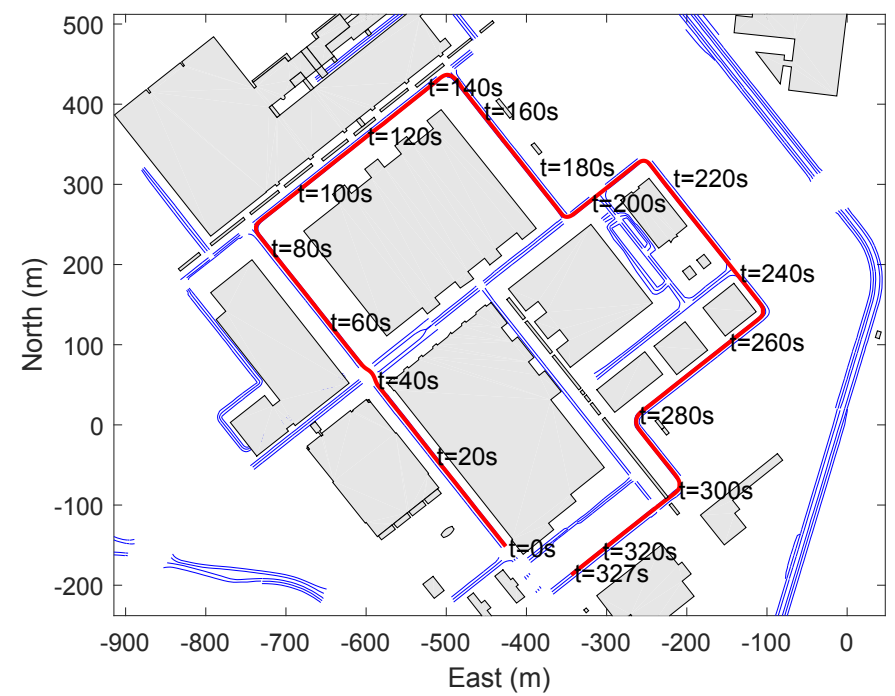

Figure 6: Test area and reference trajectory (in red) in the local ENU frame. The starting point, the point of arrival and the traveling direction are indicated by time-stamps. The lane markings are plotted in blue.

One advantage of tightly coupling GNSS is that the localization solver utilizes only the raw measurements of a high quality. As described in algorithm 1, the satellites in view are selected according to the elevation angle, the $C / N_{0}$ and validated one by one using innovation gating. Ten satellites in total were in view, but in general only five or six of them were in use at the same time (never more than eight). (Fig. 12). Some satellites (e.g. 4 and 13) were always rejected due to their low elevation angle below 15 degrees. It will also be remarked that the number of satellites used falls rapidly when the vehicle enters the urban area $(t=112-123 s)$. This is an illustration of the cautious behavior of the filter that is tuned to reject doubtful measures.

In order to appreciate the benefits of the camera we looked at what happened when it was removed from the tightly coupled filter (that we designate TC EKF). Fig. 13 shows the cumulative distribution functions of the horizontal positioning errors. The filter is clearly more accurate when the camera is included. For instance, the accuracy is four times better for the $90 \%$-percentile. Positioning performance is considerably better than standalone GNSS, even without using the camera measurement. This confirms the usefulness of data fusion with the DR sensors is very useful, and also confirms that the autoregressive modeling of the pseudorange bias is effective.

Let us now look at the estimated pseudorange biases. In each subplot of Fig. 14 the x-axis corresponds to time in seconds and the y-axis shows the biases $\varepsilon^{i}$ in meters. The bias on every pseudorange was initialized to zero with a large uncertainty. Measurements from satellites 4 and 13 were not used. Satellite 26 became available only after 100s and was lost after 200s. The estimated biases are quite smooth and stay in the order of a few meters throughout the trial, which is in line with what we expected. Fig. 15 shows the estimated $3 \sigma$ bounds for each satellite in view. The error bounds converge toward constants, which indicates an observable behavior.
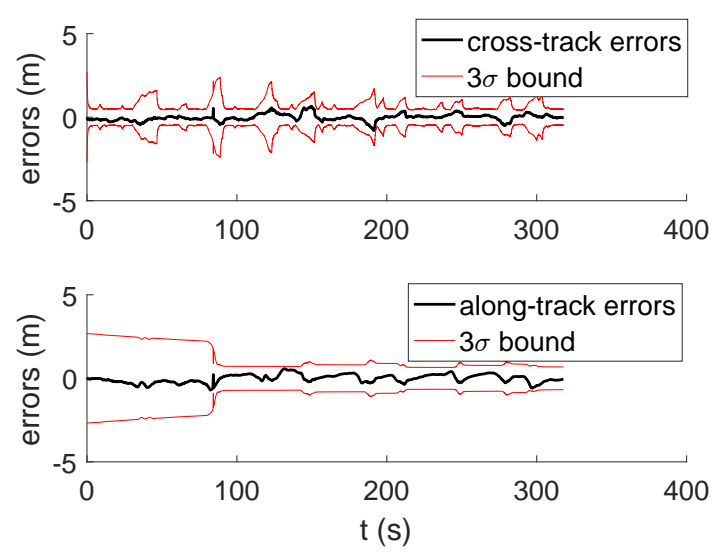

(a) Test 1
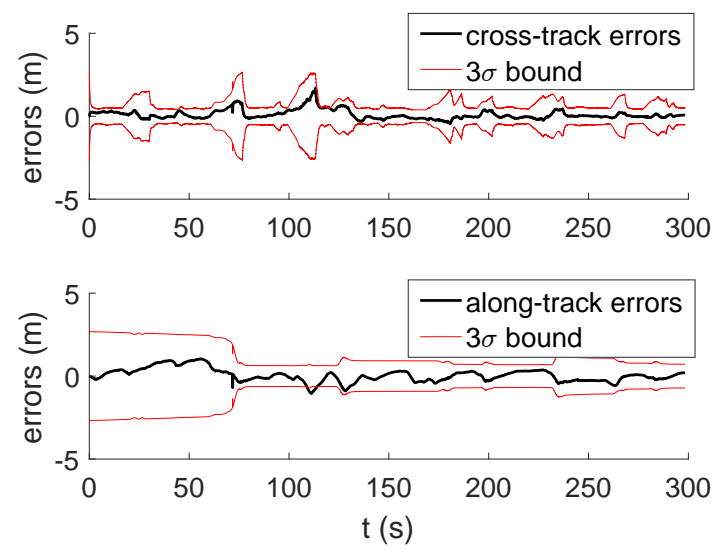

(b) Test 2
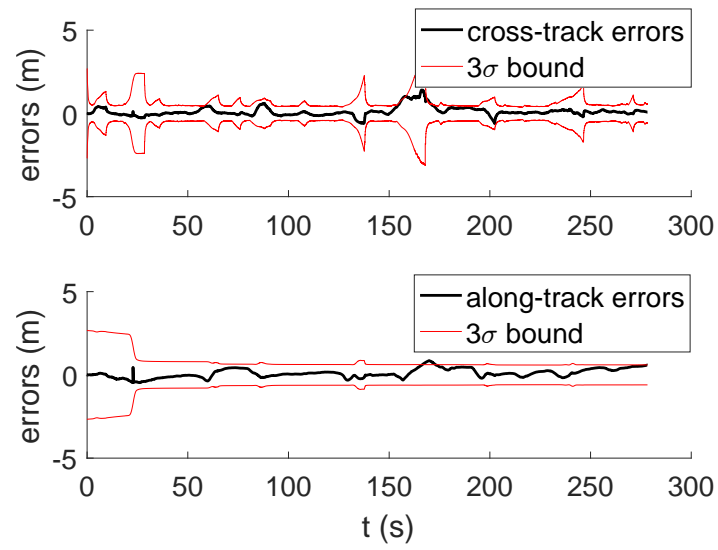

(c) Test 3

Figure 7: Along-track and cross-track positioning errors computed by the tightly coupled filter.

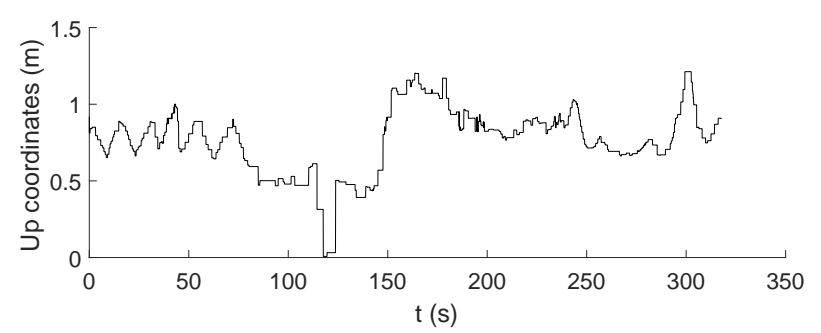

Figure 8: Altitude of the vehicle obtained from the map after mapmatching (test 1). 


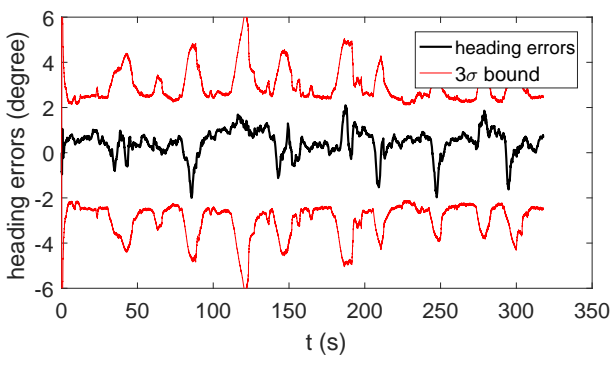

Figure 9: Heading errors (test1).

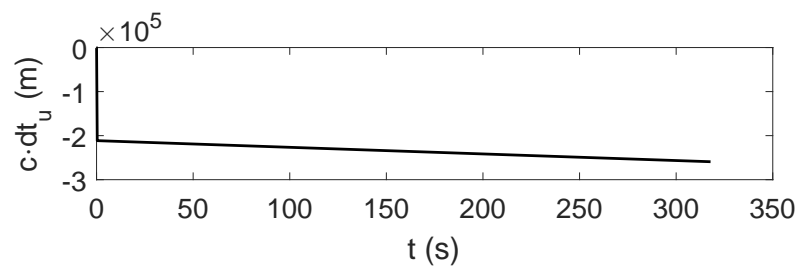

(a) Estimated clock bias times the speed of light

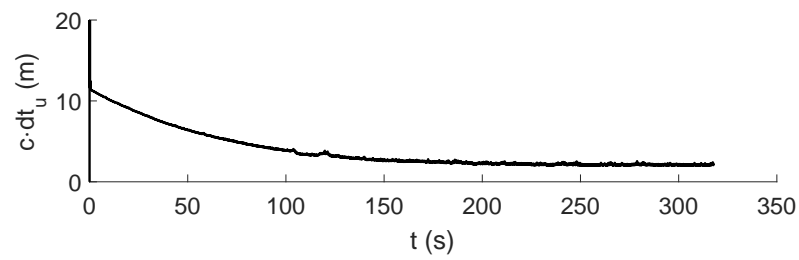

(b) Estimated $3 \sigma$ bound

Figure 10: Receiver clock bias estimates in meters (test 1).

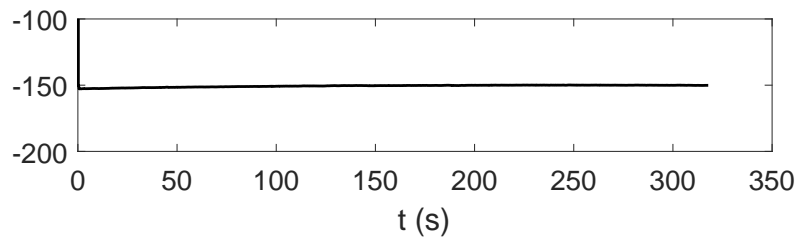

(a) Clock bias drift times the speed of light

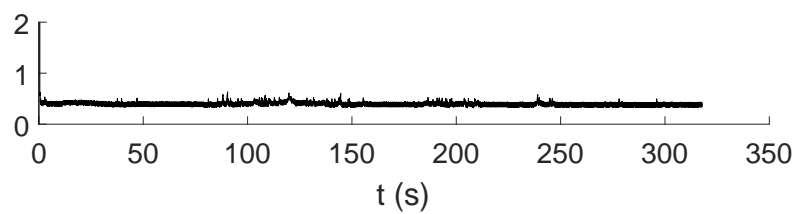

(b) Estimated $3 \sigma$ bound

Figure 11: Clock bias drift estimates in meters per second (test 1).

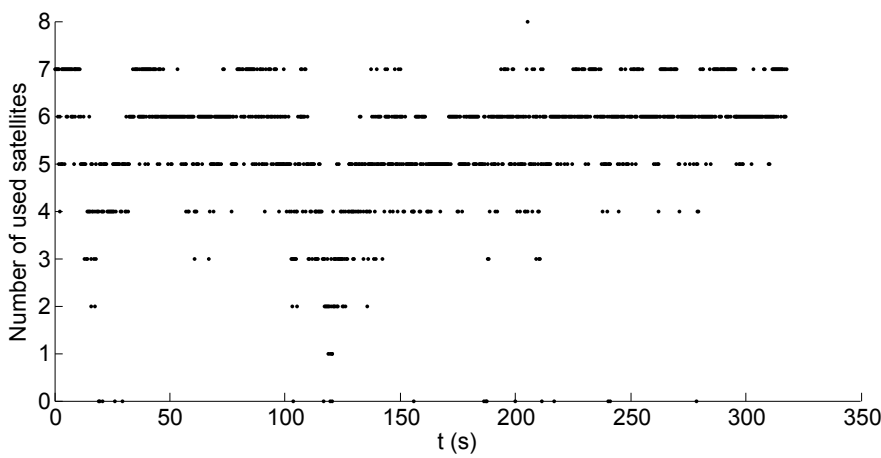

Figure 12: Number of used satellites after outlier rejection.

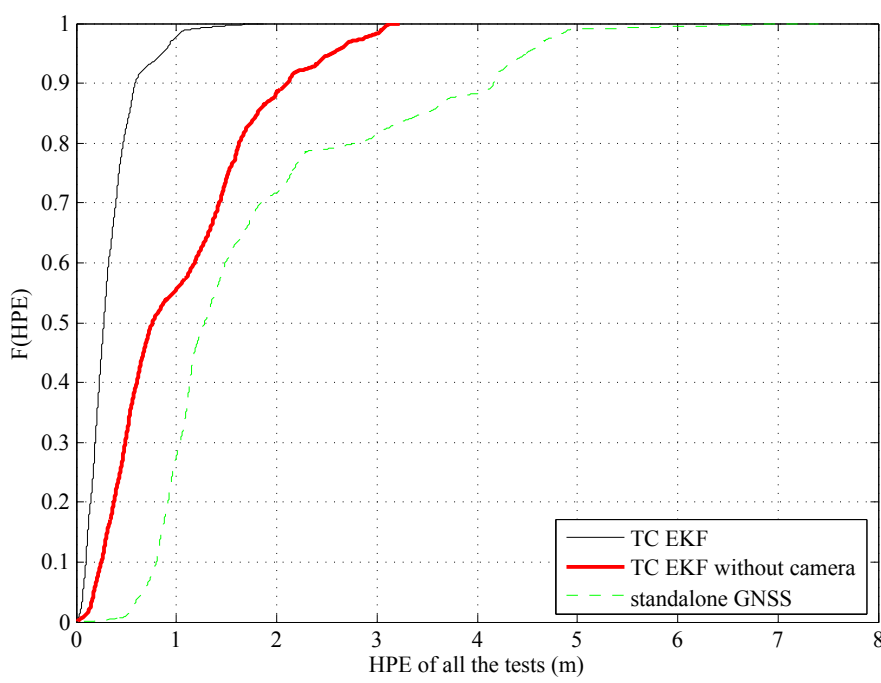

Figure 13: Plots of cumulative distribution functions $(C D F)$ of the HPEs (for the three tests).
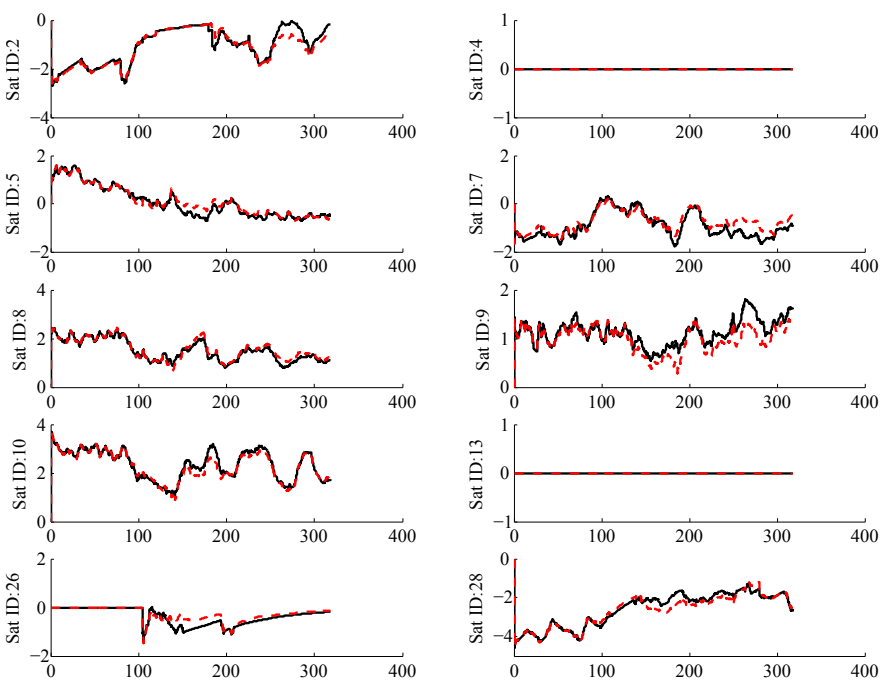

Figure 14: Estimated biases of the pseudoranges. With (red) and without (black) the camera (test 1).
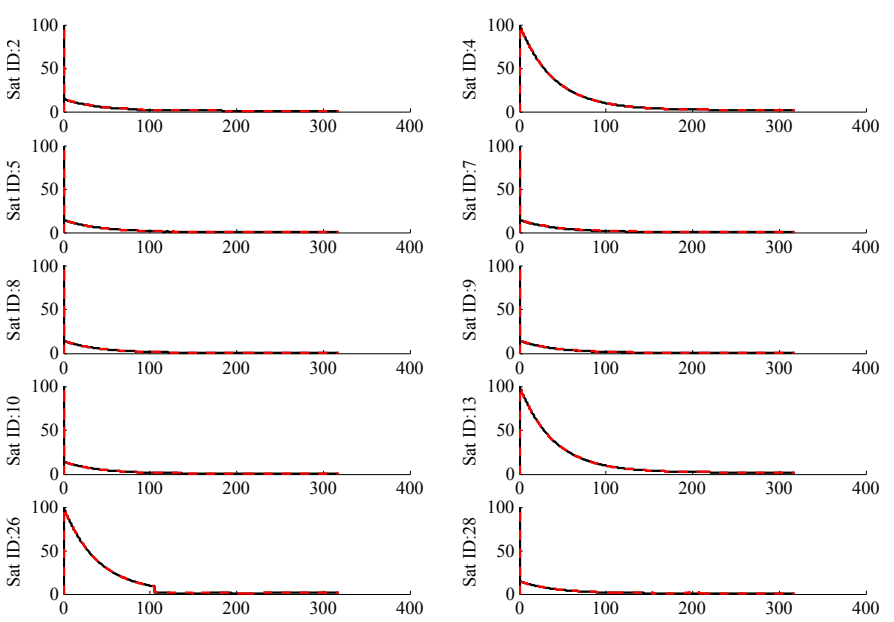

Figure 15: Estimated $3 \sigma$ bounds of the pseudorange biases. With (red) and without (black) the camera (test 1). 
We may conclude that modeling and estimating a time correlated bias on pseudorange of each satellite is feasible even without the camera. But some differences in the bias estimates with and without the camera are clearly visible (see Fig. 14). The map-based visual measures therefore have an impact on the estimation of the biases. Unfortunately, we do not have a ground truth system to assess the quality of the estimated pseudorange biases. Nevertheless, given the improvement in the positioning accuracy (Fig. 13), there is no doubt that the bias estimates are improved by the camera, which is very useful in maintaining a good estimate of the pose of the car when crossing intersections without lane markings.

\section{Consistency analysis}

As discussed in [13], Bayesian state filtering is often overconfident. We are interested in knowing whether using raw GNSS measurements has an advantage over a loosely coupled scheme with regard to the consistency of the position error.

Filter consistency can be done by analyzing the normalized positioning error (HPE) squared $e^{2}$. It is defined by the $2 \mathrm{D}$ position components of the state vector:

$$
e^{2}=\left(\begin{array}{c}
e_{x} \\
e_{y}
\end{array}\right)^{T} P_{H P E}^{-1}\left(\begin{array}{c}
e_{x} \\
e_{y}
\end{array}\right)
$$

where $P_{H P E}=\left(\begin{array}{cc}\sigma_{x}^{2} & \sigma_{x y}^{2} \\ \sigma_{x y}^{2} & \sigma_{y}^{2}\end{array}\right)$ and $\sigma_{x}, \sigma_{y}$ and $\sigma_{x y}$ are estimated by the filter. $e_{x}=\hat{x}-x_{r e f}$ and $e_{y}=\hat{y}-y_{r e f}$, with $\left(x_{r e f}, y_{r e f}\right)$ the ground truth of the vehicle horizontal position and $(\hat{x}, \hat{y})$ the estimated position.

If the assumptions in the model and the tuning filter are correct, then Eq. (23) follows a $\chi^{2}$ distribution under a Gaussian assumption with two degrees of freedom (denoted $\chi^{2}(2)$ in the following).

To examine the consistency of the horizontal positioning error, one usually looks at the percentage of samples exceeding a determined threshold $k^{2}$ given by a $\chi^{2}(2)$ distribution and corresponding to a percentile.

$$
\left(\begin{array}{c}
e_{x} \\
e_{y}
\end{array}\right)^{T} P_{H P E}^{-1}\left(\begin{array}{c}
e_{x} \\
e_{y}
\end{array}\right)>k^{2}
$$

It is easily shown that Eq. (24) is equivalent to the following:

$$
\sqrt{e_{x}^{2}+e_{y}^{2}}>k \sqrt{\frac{1}{\mathbf{u}_{\mathbf{e}}^{T} P_{H P E}^{-1} \mathbf{u}_{\mathbf{e}}}}
$$

where $\mathbf{u}_{\mathbf{e}}=\left(\begin{array}{c}e_{x} \\ e_{y}\end{array}\right) / \sqrt{e_{x}^{2}+e_{y}^{2}}$ is the unit vector supporting the horizontal positioning error .

Let us define $\sigma_{H P E}$ as the standard deviation along the horizontal positioning error vector:

$$
\sigma_{H P E}=\sqrt{\frac{1}{\mathbf{u}_{\mathbf{e}}^{T} P_{H P E}^{-1} \mathbf{u}_{\mathbf{e}}}}
$$

Fig. 16 illustrates the definition of $k \sigma_{H P E}$, where the equation of the ellipse is

$$
\left(\begin{array}{l}
x-\hat{x} \\
y-\hat{y}
\end{array}\right)^{T} P_{H P E}^{-1}\left(\begin{array}{l}
x-\hat{x} \\
y-\hat{y}
\end{array}\right)=k^{2}
$$

We have chosen to set the consistency risk to $10^{-2}(1 \%)$ which is a common choice in engineering, but this value can

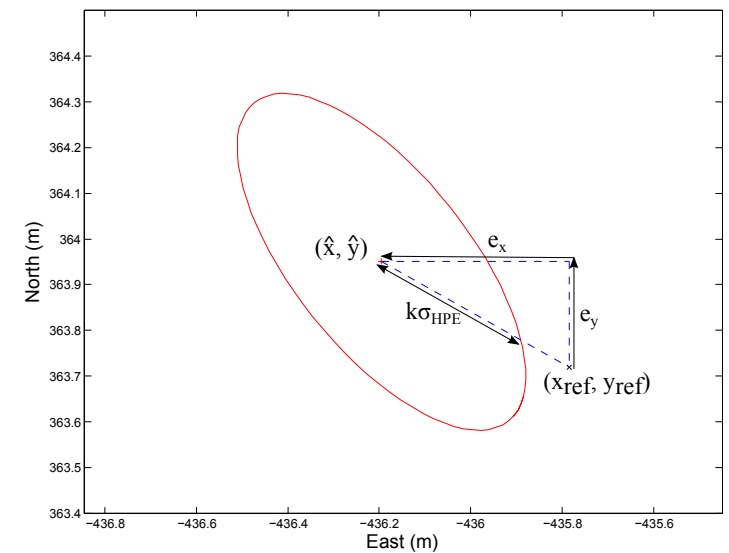

Figure 16: $k \sigma_{H P E}$ illustration. $(\hat{x}, \hat{y})$ is the estimated position and $\left(x_{r e f}, y_{r e f}\right)$ the ground truth of the horizontal position. Here, the reference is located outside of the confidence domain.

\begin{tabular}{|c|c|c|c|c|}
\hline \multirow{2}{*}{} & \multicolumn{4}{|c|}{ Consistency failure rate } \\
\cline { 2 - 5 } & test 1 & test 2 & test 3 & global \\
\hline \hline LC EKF & $41.2 \%$ & $31.8 \%$ & $47.1 \%$ & $39.9 \%$ \\
\hline TC EKF & 0 & $3.1 \%$ & $6.1 \%$ & $2.9 \%$ \\
\hline
\end{tabular}

Table II: Consistency failure rate of the two methods.

easily be adjusted to any specific requirement. According to a $\chi^{2}(2)$ distribution, $k^{2}=9.21$. In this case, the corresponding bound of the $2 \mathrm{D}$ estimated position is $3.035 \sigma_{H P E}$, with $\sigma_{H P E}$ being estimated in real-time by the filter. Therefore, consistency failure occurs when the real error is beyond this $3.035 \sigma_{H P E}$ bound, which means

$$
\sqrt{e_{x}^{2}+e_{y}^{2}}>3.035 \sigma_{H P E}
$$

We compared the consistency of the tightly coupled filter to a loosely coupled method in which the fixes computed by the GNSS receiver are fed into the navigation filter [32]. This loosely coupled implementation is designated below as $L C$ $E K F$. The parameters of this filter were set with the same values as $T C E K F$.

Fig. 17 shows the consistency of the loosely and tightly coupled methods. These histograms can be considered as simplified Stanford diagrams. The x-axis is HPE $\left(\sqrt{e_{x}^{2}+e_{y}^{2}}\right)$ and the $\mathrm{y}$-axis $3.035 \sigma_{H P E}$. The grey area corresponds to overconfident outcomes of the filters. The points in the grey area therefore satisfy the condition in Eq. (27) and the number of these points is used to compute the percentage of samples exceeding the determined threshold.

TC EKF is clearly more consistent, as the density of the points is above the first bisector. The consistencies for each test are listed in Table II. The global failure rate of $L C$ $E K F$ is $39.9 \%$ which indicates that the filter is significantly overconfident. TC EKF reduces the consistency failure rate down to $2.9 \%$ (Table II), which is in the same order as the chosen risk (1\%). The tightly coupled method therefore provides more reliable confidence domains than the loosely coupled method.

In practice, the estimated confidence is compared to a threshold to indicate use or don't use to client applications. It is 

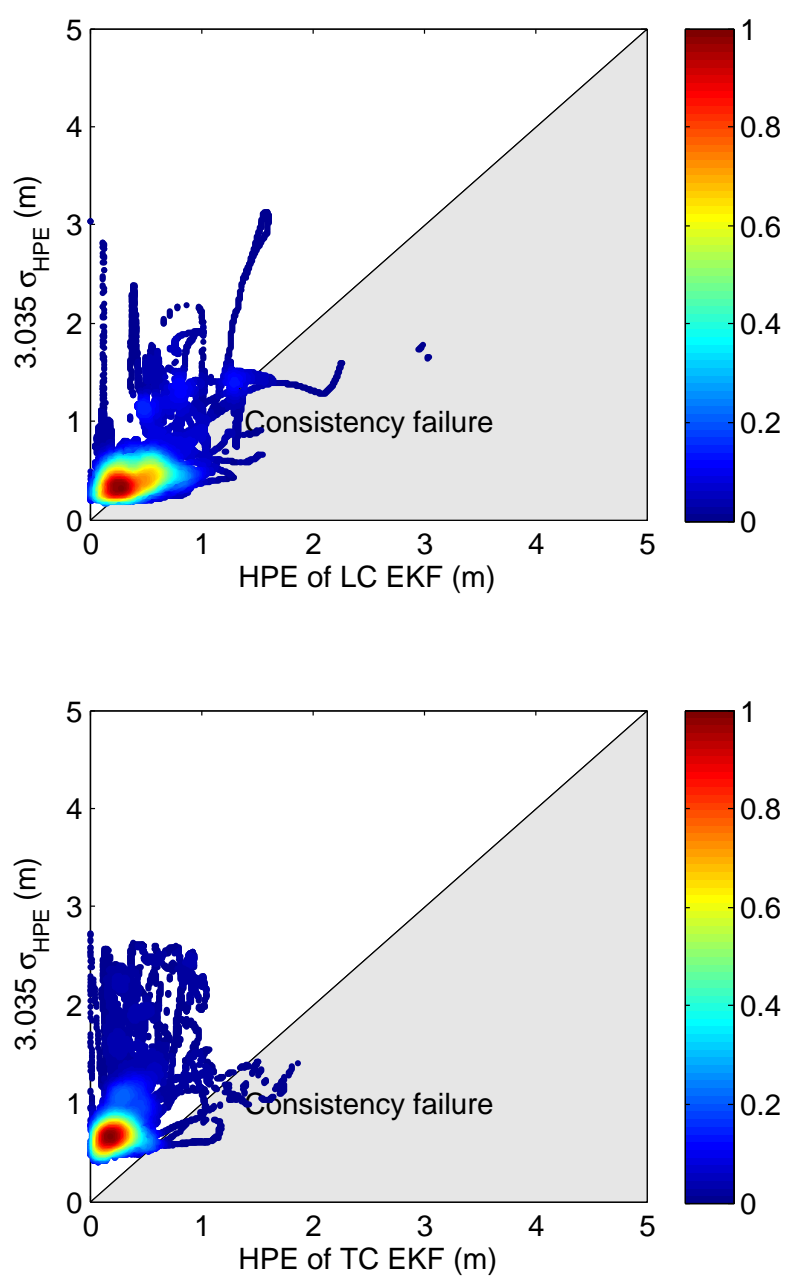

Figure 17: Consistency plots for the three tests.

\begin{tabular}{|c|c|c|c|c|}
\hline \multirow{2}{*}{} & \multicolumn{4}{|c|}{ Confidence domain size $3.035 \sigma_{H P E}(\mathrm{~m})$} \\
\cline { 2 - 5 } & median & $75^{\text {th }}$ percentile & $95^{\text {th }}$ percentile & $\max$ \\
\hline \hline LC EKF & 0.47 & 0.72 & 1.46 & 3.13 \\
\hline TC EKF & 0.78 & 1.25 & 2.47 & 2.78 \\
\hline
\end{tabular}

Table III: Confidence domain size of the two methods.

therefore important, in terms of availability of the positioning information, to provide confidence zones that are as narrow as possible.

Table III lists different statistics on the confidence domain size for both filters. The sizes are within the same order of magnitude. $L C E K F$ provides a tighter confidence domain in terms of median, $75^{t h}$ percentile and $95^{t h}$ percentile. This confirms that it is too confident. Moreover, it has a bigger confidence domain size max, which indicates that sometimes the confidence is low. We may therefore conclude that TC EKF provides a better estimate of confidence, which is important for the availability of positioning information.

\section{CONCLUSION}

In this paper, we have proposed and studied a method to merge raw GNSS measurements and lane marking measurements detected by a camera. In order to reduce the dimension of the state vector of the state space, the linear velocity and angular velocity are used as measured input and the altitude is extracted from the map. The assumption that the vehicle is traveling on a horizontal surface is made possible by the use of a navigation frame close to the navigation area. We proposed an error model to estimate the errors on the pseudoranges. A tightly coupled filter with correlated noises was developed to reduce the computational effort and improve the real-time performance. Low cost sensors were used to test the method. Outdoor experiments took place in suburban conditions with an experimental vehicle. Generally speaking, the tightly coupled method performed best in terms of accuracy and consistency, while keeping the confidence domains tight. In addition, it was able to improve the use of GNSS measurements in situations with less than four satellites in line of sight. However, the design process is more complicated than a loosely coupled approach. We consider that the tightly coupled method is the better method when the vehicle is traveling in complex GNSS environments with satellite outages and multipath propagation, because the filter is able to function with very few satellites, and it is better at excluding raw satellite measurements contaminated by multipath. In good GNSS environments, the loosely coupled method may be adequate if the requirements in terms of accuracy and consistency are less stringent.

The lane detection camera used here was designed for lane departure warning systems, and the model we used is unable to detect multiple lanes. This is an important consideration where the localization uncertainty becomes too great to distinguish different lanes that have identical markings. An accurate timestamping of the visual measurements is another important issue, particularly at higher speeds. Our method also relies on accurate lane marking maps. The widespread availability of these maps will require a reliable infrastructure for creating and maintaining them on a large scale.

\section{Acknowledgments}

This work was carried out within the framework of Equipex ROBOTEX (ANR-10- EQPX-44-01). The authors would like to thank Javier-Ibañez Guzman for his assistance and Nabil Chaari and Stéphane Bonnet for their support in the experiments.

\section{REFERENCES}

[1] G. Agamennoni, J. I. Nieto, and E. M. Nebot. An outlier-robust kalman filter. In IEEE International Conference on Robotics and Automation, pages 1551-1558, May 2011.

[2] M. Bahrami and M. Ziebart. Instantaneous doppler-aided rtk positioning with single frequency receivers. In 2010 IEEE/ION Position Location and Navigation Symposium (PLANS), pages 70-78, May 2010.

[3] Y. Bar-shalom, X. Li, and T. Kirubarajan. Estimation with Applications to Tracking and Navigation. John Wiley \& Sons, Inc., New York, NY, USA, 2002.

[4] R. Benjamin Harris and Richard G. Mach. The gpstk: an open source gps toolkit. GPS Solutions, 11(2):145-150, 2007.

[5] D. Bétaille, F. Peyret, M. Ortiz, S. Miquel, and L. Fontenay. A new modeling based on urban trenches to improve gnss positioning quality of service in cities. IEEE Intelligent Transportation Systems Magazine, 5(3):59-70, Fall 2013.

[6] D. Bétaille and R. Toledo-Moreo. Creating enhanced maps for lanelevel vehicle navigation. Intelligent Transportation Systems, IEEE Transactions on, 11(4):786-798, Dec 2010.

[7] P. Bonnifait, M. Jabbour, and G. Dherbomez. Real-time implementation of a gis-based localization system for intelligent vehicles. EURASIP Journal of Embedded Systems, vol. 2007:12-24, June 2007. 
[8] S. Brummer, F. Janda, G. Maier, and A. Schindler. Evaluation of a mapping strategy based on smooth arc splines for different road types. In Intelligent Transportation Systems - (ITSC), 2013 16th International IEEE Conference on, pages 160-165, Oct 2013.

[9] T. Chang, L. Wang, and F. Chang. A solution to the ill-conditioned gps positioning problem in an urban environment. IEEE Transactions on Intelligent Transportation Systems, 10(1):135-145, 2009.

[10] L. Chanthalansy and A. Noureldin. Carrier-to-noise density and ai for ins/gps integration. Inside GNSS, 2009(9/10):20-29, 2009.

[11] L. Conde, R. Chelim, and U. Nunes. Collaborative vehicle selflocalization using multi-gnss receivers and $\mathrm{v} 2 \mathrm{v} / \mathrm{v} 2 \mathrm{i}$ communications. In 2015 IEEE 18th International Conference on Intelligent Transportation Systems, pages 2525-2532, Sept 2015.

[12] S. Cooper and H. Durrant-Whyte. A kalman filter model for gps navigation of land vehicles. In Proceedings of the IEEE/RSJ/GI International Conference on Intelligent Robots and Systems, volume 1, pages 157-163 vol.1, Sep 1994.

[13] V. Drevelle and P. Bonnifait. Localization confidence domains via set inversion on short-term trajectory. IEEE Transactions on Robotics, 29(5):1244-1256, Oct 2013.

[14] C. Fouque and P. Bonnifait. Matching raw gps measurements on a navigable map without computing a global position. IEEE Transactions on Intelligent Transportation Systems, 13(2):887-898, June 2012.

[15] C. Fouque, P. Bonnifait, and D. Bétaille. Enhancement of global vehicle localization using navigable road maps and dead-reckoning. In Position, Location and Navigation Symposium, 2008 IEEE/ION, pages 12861291, May 2008.

[16] P. Furgale et al. Toward automated driving in cities using close-tomarket sensors: An overview of the v-charge project. In Intelligent Vehicles Symposium (IV), 2013 IEEE, pages 809-816, June 2013.

[17] P.D. Groves. Principles of GNSS, Inertial, and Multisensor Integrated Navigation Systems, Second Edition: GNSS/GPS. Artech House, 2013.

[18] U. Iqbal, J. Georgy, W.F. Abdelfatah, M.J. Korenberg, and A. Noureldin. Pseudoranges error correction in partial gps outages for a nonlinear tightly coupled integrated system. IEEE Transactions on Intelligent Transportation Systems, 14(3):1510-1525, Sept 2013.

[19] K. Jo, K. Chu, and M. Sunwoo. Gps-bias correction for precise localization of autonomous vehicles. In Intelligent Vehicles Symposium (IV), 2013 IEEE, pages 636-641, June 2013.

[20] O. Le Marchand, Ph. Bonnifait, J. Ibanez-Guzman, D. Bétaille, and F. Peyret. Characterization of gps multipath for passenger vehicles across urban environments. ATTI dell'Istituto Italiano di Navigazione, (189):77-88, 072009.

[21] B.H. Lee, J.H. Song, J.H. Im, S.H. Im, M.B. Heo, and G.I. Jee. $\mathrm{Gps} / \mathrm{dr}$ error estimation for autonomous vehicle localization. Sensors, 15(8):20779, 2015.

[22] P. S. Maybeck. Stochastic models, estimation, and control, volume 141 of Mathematics in Science and Engineering. 1979.

[23] P. Merriaux, Y. Dupuis, P. Vasseur, and X. Savatier. Fast and robust vehicle positioning on graph-based representation of drivable maps. In IEEE International Conference on Robotics and Automation, pages 2787-2793, May 2015.

[24] I. Miller, M. Campbell, and D. Huttenlocher. Map-aided localization in sparse global positioning system environments using vision and particle filtering. Journal of Field Robotics, 28(5):619-643, 2011.

[25] M. Sahmoudi N. Kbayer and E. Chaumette. Robust gnss navigation in urban environments by bounding nlos bias of gnss pseudoranges using a $3 \mathrm{~d}$ city model. In Proceedings of the 28th International Technical Meeting of The Satellite Division of the Institute of Navigation, pages 2410-2420, Sept. 2015

[26] M. Phatak and S. Kohli. Position fix from three gps satellites and altitude: a direct method. IEEE Transactions on Aerospace and Electronic Systems, 35(1):350-354, Jan 1999.

[27] A. Rabaoui, N. Viandier, E. Duflos, J. Marais, and P. Vanheeghe. Dirichlet process mixtures for density estimation in dynamic nonlinear modeling: Application to gps positioning in urban canyons. IEEE Transactions on Signal Processing, 60(4):1638-1655, April 2012.

[28] J. Rife. Collaborative vision-integrated pseudorange error removal: Team-estimated differential gnss corrections with no stationary reference receiver. IEEE Transactions on Intelligent Transportation Systems, 13(1):15-24, March 2012.

[29] C. Rose, J. Britt, J. Allen, and D. Bevly. An integrated vehicle navigation system utilizing lane-detection and lateral position estimation systems in difficult environments for gps. IEEE Transactions on Intelligent Transportation Systems, 15(6):2615-2629, Dec 2014.
[30] M. Schreiber, C. Knoppel, and U. Franke. Laneloc: Lane marking based localization using highly accurate maps. In Intelligent Vehicles Symposium (IV), 2013 IEEE, pages 449-454, June 2013.

[31] J. Snider. Automatic Steering Methods for Autonomous Automobile Path Tracking. Technical report Carnegie Mellon University Robotics Institute CMU-RI-TR-09-08, 2009.

[32] Z. Tao and P. Bonnifait. Tightly coupling gps with lane markings for autonomous vehicle navigation. In IEEE International Conf. on Intelligent Transportation Systems, pages 439-444, Oct 2014.

[33] Z. Tao and P. Bonnifait. Road invariant extended kalman filter for an enhanced estimation of gps errors using lane markings. In IEEE/RSJ International Conference on Intelligent Robots and Systems, pages 3119-3124, Hamburg, Germany, Sept. 2015.

[34] Z. Tao, P. Bonnifait, V. Frémont, and J. Ibañez-Guzman. Mapping and localization using gps, lane markings and proprioceptive sensors. In 2013 IEEE/RSJ International Conference on Intelligent Robots and Systems, pages 406-412, Nov 2013.

[35] G. Taylor, G. Blewitt, D. Steup, S. Corbett, and A. Car. Road reduction filtering for gps-gis navigation. Transactions in GIS, 5(3):193-207, 2001.

[36] A. Vu, A. Ramanandan, A. Chen, J.A. Farrell, and M. Barth. Realtime computer vision/dgps-aided inertial navigation system for lane-level vehicle navigation. IEEE Transactions on Intelligent Transportation Systems, 13(2):899-913, June 2012.

[37] A. Wieser, M. Gaggl, and H. Hartinger. Improved positioning accuracy with high-sensitivity gnss receivers and snr aided integrity monitoring of pseudo-range observations. In International Technical Meeting of the Satellite Division of The Institute of Navigation (ION GNSS), pages 1545-1554, Sept. 2005.

[38] S. Zair, S. Le Hegarat-Mascle, and E. Seignez. A-contrario modeling for robust localization using raw gnss data. IEEE Transactions on Intelligent Transportation Systems, 17(5):1354-1367, May 2016.

[39] J. Ziegler et al. Making bertha drive-an autonomous journey on a historic route. IEEE Intelligent Transportation Systems Magazine, 6(2):8-20, 2014.

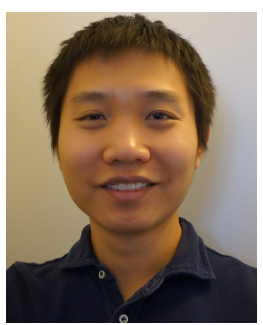

Zui Tao received his B.Sc. degree in vehicle engineering from Jilin University, Jilin, China, in 2010, and his M.Sc. and Ph.D. degrees in computer science from Sorbonne Universités, Université de Technologie de Compiègne, Compiègne, France, in 2012 and 2016 respectively. His research interests include state estimation, sensor fusion and localization systems for autonomous driving. He is currently working as postdoctoral researcher at the VEDECOM Institute, France, towards embedding more clean energy and intelligence into modern automobiles.

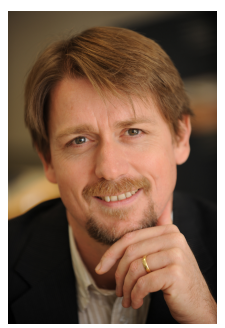

Philippe Bonnifait is a professor in the Computer Science and Engineering department of the Université de Technologie de Compiègne (UTC) in France. He obtained his Ph.D. in automatic control and computer science at the École Centrale de Nantes in 1997. Since 1998, he has been with Heudiasyc UMR 7253, a joint research unit between UTC and CNRS. His research interests are Intelligent Vehicles, and high-integrity positioning and mapmatching for autonomous navigation in structured outdoor environments. 